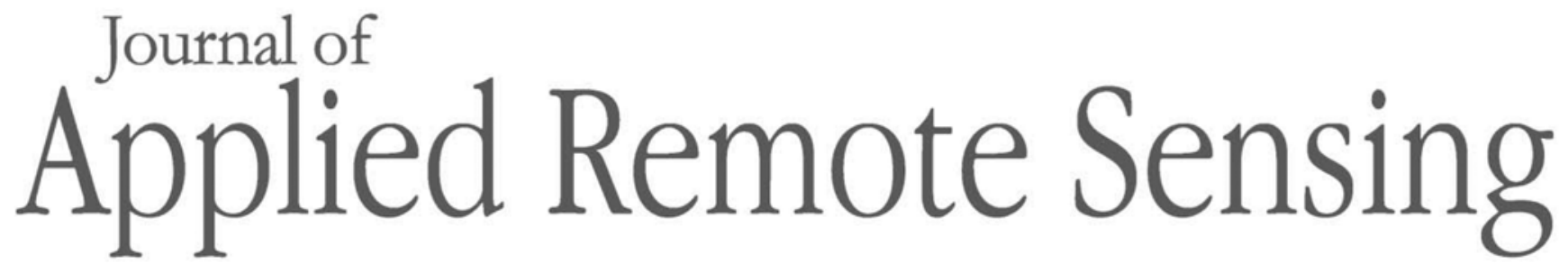

RemoteSensing.SPIEDigitalLibrary.org

\title{
Polarimetric synthetic aperture radar application for tropical peatlands classification: a case study in Siak River Transect, Riau Province, Indonesia
}

Dandy Aditya Novresiandi

Ryota Nagasawa 


\title{
Polarimetric synthetic aperture radar application for tropical peatlands classification: a case study in Siak River Transect, Riau Province, Indonesia
}

\author{
Dandy Aditya Novresiandia,* and Ryota Nagasawa ${ }^{\mathrm{b}}$ \\ ${ }^{a}$ Tottori University, United Graduate School of Agricultural Sciences, Tottori, Japan \\ ${ }^{\mathrm{b}}$ Tottori University, Faculty of Agriculture, Tottori, Japan
}

\begin{abstract}
Mapping spatial distributions of tropical peatlands is important for properly estimating carbon emissions and for providing information that aids in the sustainable management of tropical peatlands, particularly in Indonesia. This study evaluated the performance of phased array type L-band synthetic aperture radar (SAR) (PALSAR) dual-polarization and fully polarimetric data for tropical peatlands classification. The study area was in Siak River Transect, Riau Province, Indonesia, a rapidly developing region, where the peatland has been intensively converted mostly into oil palm plantations over the last two decades. Thus, polarimetric features derived after polarimetric decompositions, backscatter coefficients measurements, and the radar vegetation index were evaluated to classify tropical peatlands using the decision tree classifier. Overall, polarimetric features generated by the combination of dual-polarization and fully polarimetric data yielded an overall accuracy (OA) of $69 \%$ and a kappa coefficient (K) of 0.57. The integration of an additional feature, "distance to river," to the algorithm increased the OA to $76 \%$ and $\mathrm{K}$ to 0.66 . These results indicated that the methodology in this study might serve as an efficient tool in tropical peatlands classification, especially when involving the use of L-band SAR dual-polarization and fully polarimetric data. (C) The Authors. Published by SPIE under a Creative Commons Attribution 3.0 Unported License. Distribution or reproduction of this work in whole or in part requires full attribution of the original publication, including its DOI. [DOI: 10 .1117/1.JRS.11.016040]
\end{abstract}

Keywords: decision tree classifier; dual-polarization data; fully polarimetric data; PALSAR.

Paper 16968 received Dec. 19, 2016; accepted for publication Mar. 8, 2017; published online Mar. 25, 2017.

\section{Introduction}

In recent years, there has been considerable interest in the potential of tropical peatlands as carbon storage, as well as the magnitude of their carbon emissions and their important role in climate change processes. These concerns should be responded to via an accurate inventory of tropical peatlands to obtain a better understanding of tropical peatlands management. ${ }^{1}$ The accurate inventory of tropical peatlands, such as in mapping spatial distributions of tropical peatlands, is important for properly estimating carbon emissions, for appropriately evaluating the effect of the land use/cover change due to rapid economic development, and for providing information that aids in the sustainable management of tropical peatlands, particularly in Indonesia. ${ }^{2,3}$ However, tropical peatlands cover relatively large areas and are primarily located in remote areas that are difficult to access. Therefore, it is challenging to map their spatial distributions. ${ }^{4}$

Remote sensing is the most effective tool for mapping spatial distributions of tropical peatlands at various spatial and temporal scales, especially when combined with ground truth data. ${ }^{3}$ Thus, the remote sensing technique serves as an advantageous tool due to its periodic monitoring system at a wide-scale synoptic view, particularly in remote sites. ${ }^{5}$ Furthermore, the growing availability of the synthetic aperture radar (SAR)-based remote sensing satellites has introduced a new prospect that allows continuous monitoring and cloud-free observations in humid tropical regions, ${ }^{6}$ that can be used for mapping spatial distributions of tropical peatlands. Previous

*Address all correspondence to: Dandy Aditya Novresiandi, E-mail: dandyaditya@gmail.com 
studies $^{7,8}$ have evaluated the potential of L-band SAR fully polarimetric data for peatlands detection and delineation in the boreal regions. Another study ${ }^{9}$ examined the combination of X-band SAR dual-polarization data and optical data for discriminating tropical peatlands in Central Kalimantan, Indonesia. An earlier report ${ }^{10}$ used the L-band SAR fully polarimetric data for evaluating the radar scattering mechanism on tropical peatlands in Central Kalimantan, Indonesia. Another report ${ }^{11}$ applied the combination of L-band SAR dual-polarization data, optical data, and digital elevation model (DEM)-derived data for mapping spatial distributions of tropical peatlands in the Cuvette Centrale, Congo Basin. However, detailed information is lacking on the performance of L-band SAR dual-polarization and fully polarimetric data for mapping spatial distributions of tropical peatlands.

The L-band SAR dual-polarization data include two channels-horizontal-horizontal (HH) and horizontal-vertical (HV) — of polarization, whereas the fully polarimetric data comprise four channels- $\mathrm{HH}, \mathrm{HV}$, vertical-horizontal $(\mathrm{VH})$, and vertical-vertical $(\mathrm{VV})$-of polarization. These data operated in $1.27 \mathrm{GHz}$, permitting the cloud penetration ability and reported to be capable of passing through a certain level of vegetation cover to verify the underlying soil characteristics. ${ }^{12,13}$ These potentials make L-band SAR dual-polarization and fully polarimetric data particularly promising for use in tropical peatlands mapping activities.

Thus, in this study, the performance of phased array type L-band SAR (PALSAR) dualpolarization and fully polarimetric data was evaluated for tropical peatlands classification. The performance of the data when utilized as single usage (e.g., only dual-polarization data or only fully polarimetric data), combined (i.e., the combination of dual-polarization and fully polarimetric data), and integrated with an additional feature "distance to river" (i.e., added the topographic-derived feature "distance to river" to the dual-polarization and fully polarimetric data combination) were compared and investigated. Thus, polarimetric features derived after polarimetric decompositions (PDs), backscatter coefficients measurements, and the radar vegetation index (RVI) were evaluated to classify tropical peatlands using the decision tree classifier. In addition, the seasonal variation of tropical peatlands from the viewpoint of L-band SAR dual-polarization data was analyzed to increase the state of knowledge of tropical peatlands for classification. The findings of this study could aid in improving the state of knowledge in tropical peatlands classification, especially when involving the use of L-band SAR dual-polarization and fully polarimetric data.

\section{Materials and Methods}

\subsection{Study Area and Data}

The study area, $5 \mathrm{~km} \times 10 \mathrm{~km}$ in size, was in Siak River Transect, Riau Province, Indonesia (Fig. 1), a rapidly developing region where the peatland has been intensively converted mostly

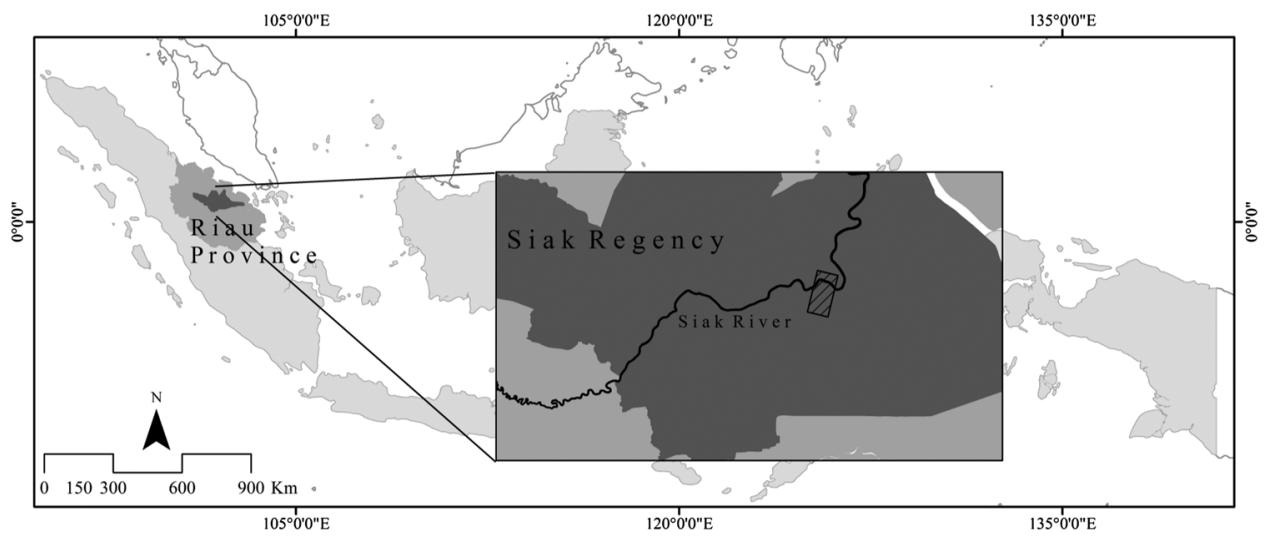

Fig. 1 Map of Indonesia showing the location of the study area (hatched rectangle). 
into oil palm plantations over the last two decades. ${ }^{14}$ Generally, this area has a flat topography with a surface elevation ranging from 2 to $10 \mathrm{~m}$ above sea level. The temperature of this area is $26.2^{\circ} \mathrm{C}$ per year on average. The annual rainfall of this area varies from 2200 to $2600 \mathrm{~mm}$ per year, with the lowest and highest monthly rainfall in July (around $110 \mathrm{~mm}$ per month) and November (around $290 \mathrm{~mm}$ per month), respectively.

In this study, two scenes of PALSAR dual-polarization data and a single scene of PALSAR fully polarimetric data were used as the primary data. The dual-polarization data were taken during the driest month of the year (ALPSRP236980000, coded as dry scene) and the wettest month of the year (ALPSRP257110000, coded as wet scene), allowing the seasonal variation analysis of tropical peatlands from the viewpoint of L-band dual-polarization SAR. The single scene of fully polarimetric data (ALPSRP175860000) was taken during the average monthly rainfall.

Moreover, Landsat 5 Thematic Mapper (TM) data were used to update the existing land use/ cover map by means of visual interpretation and to select the training samples for classification purposes. A total of 2400 points were derived for training (1680 points) the algorithm of decision tree classification and for testing (720 points) the accuracy of the classification results (Fig. 2). An existing peatland distribution map and a topographic map provided by the Indonesian Agency for Agricultural Research and Development (IAARD) were used as reference maps. In addition, a joint ground truth survey with the IAARD was conducted on May 19-22, 2015 to provide basic information about the study area. The list of data used for analysis is shown in Table 1.

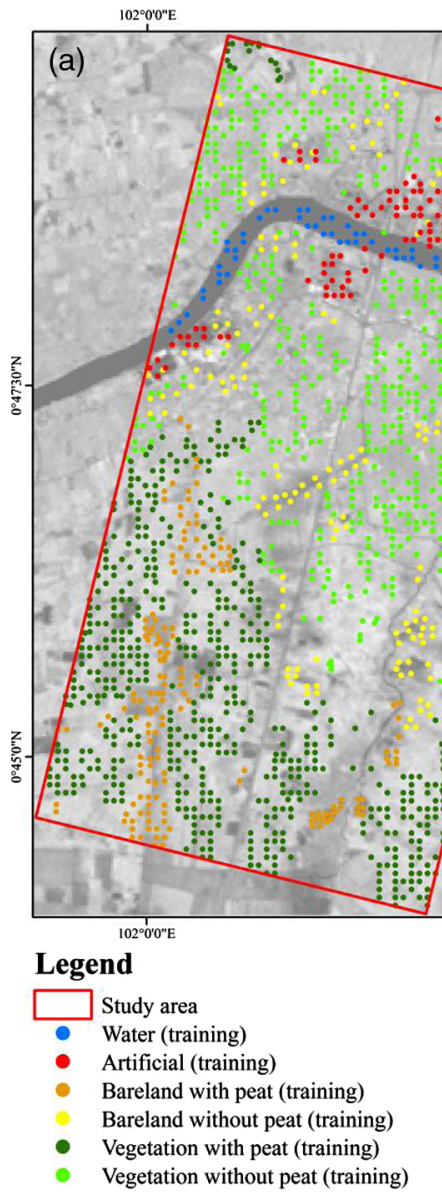

$102^{\circ} 2^{\prime} 30^{\prime \prime} \mathrm{E}$

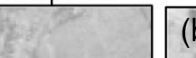

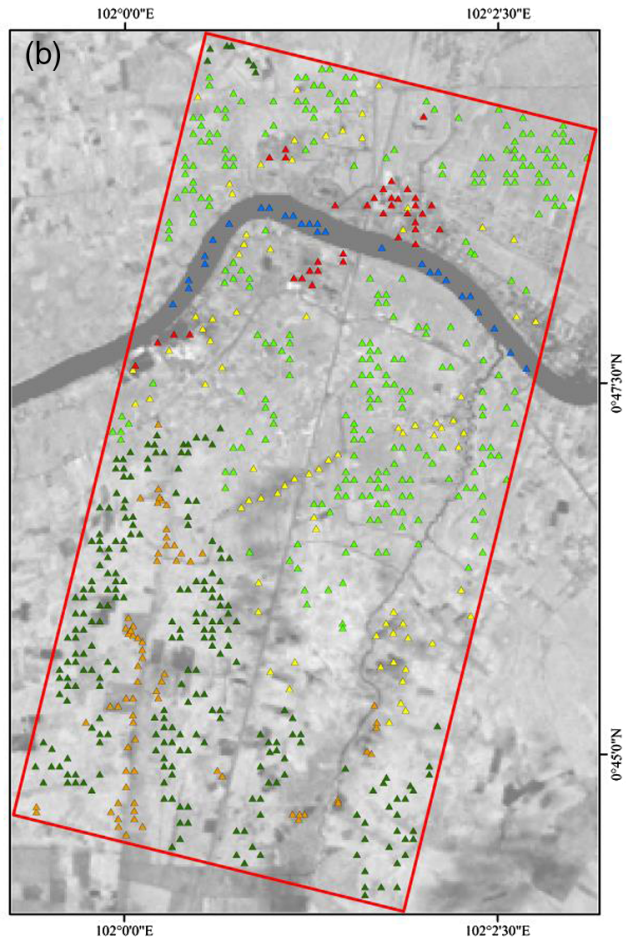

$102^{\circ} 0^{\circ} 0^{\prime \prime}$

Fig. 2 Landsat 5 image of the study area showing the spatial distribution of (a) training and (b) testing points.

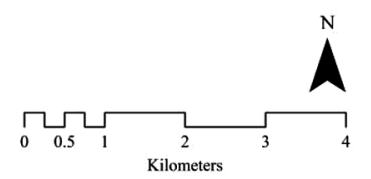


Novresiandi and Nagasawa: Polarimetric synthetic aperture radar application for tropical...

Table 1 List of data used in this study.

\begin{tabular}{llll}
\hline \hline Data usage & \multicolumn{1}{c}{ Data type } & Data ID & Acquisition date \\
\hline Primary data & PALSAR dual-polarization (dry scene) & ALPSRP236980000 & July 6, 2010 \\
& PALSAR dual-polarization (wet scene) & ALPSRP257110000 & November 21, 2010 \\
& PALSAR fully polarimetric data & ALPSRP175860000 & May 13, 2009 \\
Secondary data & Landsat 5 TM & LT51260592010033BKT00 & February 2, 2010 \\
& Landsat 5 TM & LT51260602010289BKT00 & October 16, 2010 \\
& ASTER GDEM & ASTGTM2 (Version 2) & October 17, 2011 \\
& & & (Released) \\
\hline \hline
\end{tabular}

\subsection{Data Preprocessing}

PALSAR data were imported to the PolSARPro Software for image processing. Thus, these data were radiometrically calibrated and multilooked one time in range and five times in azimuth direction for the dual-polarization data, and one time in range and seven times in azimuth direction for the fully polarimetric data. Speckle noise was reduced by applying a $7 \times 7$ Lee-refined filter, followed by conversions to the scattering matrix (S2) and a $2 \times 2$ covariance matrix (C2) for the dual-polarization data, and to the $S 2$, coherency matrix (T3) and a $3 \times 3$ covariance matrix (C3) for the fully polarimetric data. These matrices were used as inputs for deriving polarimetric features. PALSAR data were then geocoded using the advanced spaceborne thermal emission and reflection radiometer (ASTER) global digital elevation model (GDEM) to the UTM Zone $48 \mathrm{~N}$ map projection.

\subsection{Polarimetric Features for Dual-Polarization Data}

Polarimetric features for dual-polarization data were derived after PDs and backscatter coefficients were obtained. The Cloude-Pottier decomposition, ${ }^{15}$ an eigenvalue/eigenvector-based decomposition, was performed for the dual-polarization data to derive three features, viz. entropy (coded as dFBD_H for the dry-scene data and wFBD_H for the wet-scene data), anisotropy (coded as dFBD_A for the dry-scene data and wFBD_A for the wet-scene data), and alpha angle (coded as $\mathrm{dFBD} \alpha$ for the dry-scene data and $\operatorname{wFBD} \alpha$ for the wet-scene data). Two features represent the channels of polarization derived from the dual-polarization data, i.e., $\mathrm{HH}$ (coded as dFBD_HH for the dry-scene data and wFBD_HH for the wet-scene data) and HV (coded as dFBD_HV for the dry-scene data and wFBD_HV for the wet-scene data). Each scene of the dual-polarization data (wet and dry scenes) derived five polarimetric features. Thus, a total of 10 polarimetric features were computed using the dual-polarization data for the analysis carried out in this study.

\subsection{Polarimetric Features for Fully Polarimetric Data}

Polarimetric features for fully polarimetric data were derived after PDs, backscatter coefficients measurements, and the RVI were obtained. Three PDs-Cloude-Pottier decomposition, ${ }^{15}$ Freeman-Durden three-component decomposition, ${ }^{16}$ and Yamaguchi four-component decomposition ${ }^{17}$ - were applied to generate polarimetric features for the fully polarimetric data. Cloude-Pottier decomposition was also performed for the fully polarimetric data and generated three features, namely, entropy (PLR_H), anisotropy (PLR_A), and the alpha angle (PLR_ $\alpha$ ). The Freeman-Durden decomposition, a physical scattering model-based decomposition, models the covariance matrix (C3) as the contribution of three component scattering mechanisms_-surface (f_odd), double-bounce (f_double), and volume (f_volume) scattering mechanisms. The Yamaguchi decomposition scheme incorporates the nonreflection symmetry condition of copolarization and cross-polarization radar channels thus the covariance matrix (C3) is modeled as four component scattering mechanisms' contributions-surface (y_odd), 
double-bounce (y_double), volume (y_volume), and helix (y_helix) scattering mechanisms. Four features representing the channels of polarization were derived from the fully polarimetric data, i.e., HH (coded as PLR_HH), HV (coded as PLR_HV), VH (coded as PLR_VH), and VV (coded as PLR_VV).

Three features representing a decomposition approach based on the Pauli matrix were generated. ${ }^{12,15}$ These features are $\mathrm{HH}+\mathrm{VV}, \mathrm{HH}-\mathrm{VV}$, and $2 \mathrm{HV}$, associated with surface scattering, double-bounce scattering, and volume scattering, respectively. A depolarization ratio (HV/HH) was calculated that indicated the level of volume scattering. ${ }^{12}$ In addition, the total scattering power (SPAN) in four polarizations of the fully polarimetric data was generated. The SPAN is an average of $\mathrm{HH}, \mathrm{HV}+\mathrm{VH}$, and $\mathrm{VV}$, and thus has a lower speckle noise than those polarization channels individually. ${ }^{18}$ Concurrently, the RVI was derived for quantifying the amount of biomass presented in each pixel. ${ }^{19}$ The equation for the RVI is

$$
\mathrm{RVI}=\frac{8 \sigma_{\mathrm{HV}}^{0}}{\sigma_{\mathrm{HH}}^{0}+\sigma_{\mathrm{VV}}^{0}+2 \sigma_{\mathrm{HV}}^{0}},
$$

where $\sigma^{0}$ is the backscatter coefficient in linear units. This feature generally ranges between 0 and 1 and increases with the vegetation cover. Therefore, a total of 20 polarimetric features were computed from the fully polarimetric data for the analysis carried out in this study. The list of polarimetric features used for analysis is shown in Table 2 .

\subsection{Image Classification}

To evaluate the performance of PALSAR dual-polarization and fully polarimetric data in classifying tropical peatlands, polarimetric features derived by four combinations of data were used in this study to apply to the algorithm of decision tree classification: (i) the combination of the two scenes of dual-polarization data, (ii) the single scene of the fully polarimetric data, (iii) the combination of the two scenes of dual-polarization data and the single scene of the fully polarimetric data, and (iv) the combination of the two scenes of dual-polarization data, the single scene of the fully polarimetric data, and the additional feature "distance to river." These combinations of data were applied in this study to assess the capabilities of each type of data and their combinations in classifying tropical peatlands. Moreover, the integration of the additional feature "distance to river" was meant to evaluate the effectiveness of geographic information system (GIS) data integration in the SAR-based tropical peatlands classification. The feature "distance to river" was derived from a GIS data processing, which buffered the center line of the main river stream into three categories, namely, "less than $2 \mathrm{~km}$ from river," " 2 to $5 \mathrm{~km}$ from river," and "more than $5 \mathrm{~km}$ from river." Previous studies ${ }^{4,20,21}$ have found "distance to river" to be an important topographic feature in estimating the peat depth as well as in discriminating spatial distributions of tropical peatlands.

In this study, the decision tree classifier was used for classifying tropical peatlands. According to the concept of decision tree classification, ${ }^{22}$ the decision tree is a procedure of classification that recursively partitions a set of data into smaller subdivisions based on a set of criteria determined at each branch in the tree. The decision tree classifier was characterized as strictly nonparametric and required no-assumptions regarding the distributions of the input data. Moreover, the design of classification is explicit and easy to understand. The main advantages of the decision tree classifier are its ability to handle complex relations among class properties, its computational speed, and its capability to handle data represented on different measurement scales. ${ }^{23}$ Thus, the decision tree is an efficient tool for land cover classification purposes. ${ }^{24}$ Furthermore, the distance factor (DF) was used in this study to evaluate the effectiveness of a feature for separating the class pairs by calculating the distance between the different class mean values compared with the standard deviations. Hence, if the distance between the different class mean values is large compared with the standard deviations, classes are said to be well separated following the concept of feature separation. ${ }^{25}$ The expression for the DF is

$$
\mathrm{DF}_{i j}=\frac{\left|\bar{x}_{i}-\bar{x}_{j}\right|}{\sigma_{i}+\sigma_{j}}
$$


Novresiandi and Nagasawa: Polarimetric synthetic aperture radar application for tropical...

Table 2 List of polarimetric features derived for analysis.

\begin{tabular}{|c|c|c|c|}
\hline Source & Methods & Feature name & Code name \\
\hline \multirow[t]{20}{*}{ Fully polarimetric data } & Backscatter coefficients & $\mathrm{HH}$ & PLR_HH \\
\hline & & HV & PLR_HV \\
\hline & & $\mathrm{VH}$ & PLR_VH \\
\hline & & VV & PLR_VV \\
\hline & Cloude-Pottier decomposition & Entropy & PLR_H \\
\hline & & Anisotropy & PLR_A \\
\hline & & Alpha & PLR $\_\alpha$ \\
\hline & Freeman-Durden decomposition & Surface scattering & f_odd \\
\hline & & Double-bounce scattering & f_double \\
\hline & & Volume scattering & f_volume \\
\hline & Yamaguchi decomposition & Surface scattering & y_odd \\
\hline & & Double-bounce scattering & y_double \\
\hline & & Volume scattering & y_volume \\
\hline & & Helix scattering & y_helix \\
\hline & Total scattering power & SPAN & SPAN \\
\hline & $\begin{array}{l}\text { Backscatter coefficients } \\
\text { measurements (Pauli Matrix) }\end{array}$ & Surface scattering & $\mathrm{HH}+\mathrm{VV}$ \\
\hline & & Double-bounce scattering & $\mathrm{HH}-\mathrm{VV}$ \\
\hline & & Volume scattering & $2 \mathrm{HV}$ \\
\hline & $\begin{array}{l}\text { Backscatter coefficients } \\
\text { measurements }\end{array}$ & Depolarization ratio & $\mathrm{HV} / \mathrm{HH}$ \\
\hline & $\mathrm{RVI}$ & $\mathrm{RVI}$ & RVI \\
\hline \multirow{5}{*}{$\begin{array}{l}\text { Dry-scene } \\
\text { dual-polarization data }\end{array}$} & Backscatter coefficients & $\mathrm{HH}$ & dFBD_HH \\
\hline & & HV & dFBD_HV \\
\hline & Cloude-Pottier decomposition & Entropy & dFBD_H \\
\hline & & Anisotropy & dFBD_A \\
\hline & & Alpha & $\mathrm{dFBD} \alpha$ \\
\hline \multirow{5}{*}{$\begin{array}{l}\text { Wet-scene } \\
\text { dual-polarization data }\end{array}$} & Backscatter coefficients & $\mathrm{HH}$ & wFBD_HH \\
\hline & & HV & wFBD_HV \\
\hline & Cloude-Pottier decomposition & Entropy & wFBD_H \\
\hline & & Anisotropy & wFBD_A \\
\hline & & Alpha & wFBD $\alpha$ \\
\hline
\end{tabular}

where $\bar{x}$ and $\sigma$ are the mean values and standard deviations, respectively. The quality of the separation between classes $i$ and $j$ is represented by the value of $\mathrm{DF}_{i j}$. Values of $\mathrm{DF}_{i j}$ of less than 0.8 are considered below average, between 0.8 and 1.5 average, and more than 2.0 close to the complete separation of class pairs. ${ }^{26}$

In this study, six classes were selected to represent the common land use/cover on the study area from the viewpoint of L-band SAR data. These classes are water, artificial, bareland with 


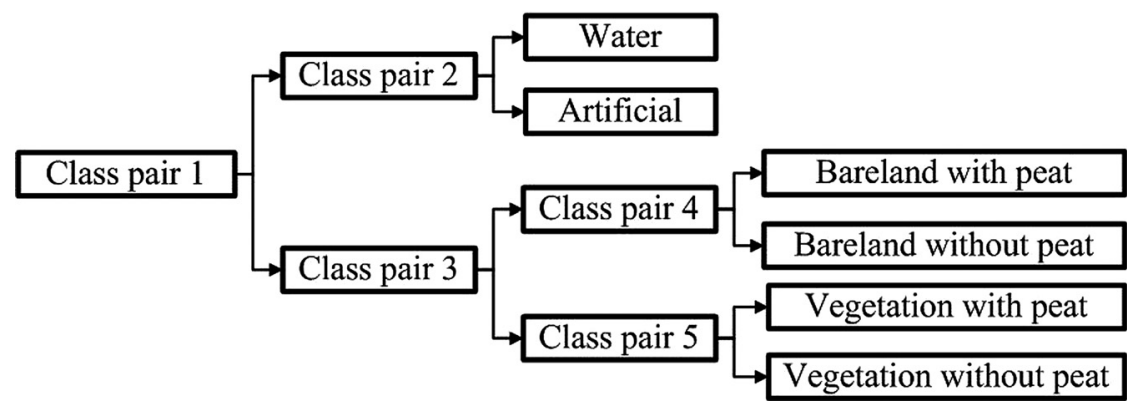

Fig. 3 The algorithm of the decision tree classification.

peat, bareland without peat, vegetation with peat, and vegetation without peat. Hence, to apply the concept of feature separation on the algorithm of the decision tree classification, five combinations of class pairs were specified: (1) ("water" plus "artificial") and others ("bareland with peat," "bareland without peat," "vegetation with peat," plus "vegetation without peat"), (2) "water" and "artificial," (3) ("bareland with peat" plus "bareland without peat") and ("vegetation with peat" plus "vegetation without peat"), (4) "bareland with peat" and "bareland without peat," and (5) "vegetation with peat" and "vegetation without peat." These class pairs were then applied to the algorithm of the decision tree classification to classify each land use/ cover class, as shown in Fig. 3.

Thus, polarimetric features with a relatively high DF on each class pair were analyzed and applied to the algorithm for decision tree classification, then the classification results' accuracy was assessed using the available testing points. Features that increase the accuracy of the classification results were then added to the algorithm and vice versa. The confusion matrix was used to perform the accuracy assessment on the classification results by means of deriving the accuracy indicators, namely, producer's accuracy (PA), user's accuracy (UA), overall accuracy $(\mathrm{OA})$, and kappa coefficient $(\mathrm{K})$. These indicators were used to assess the quality of the classification results.

\section{Results and Discussion}

\subsection{Class Pairs' Separability}

Table 3 shows the separability of five class pairs by each polarimetric feature, as measured by the DF. An italic value indicates the low performance of a feature in separating class pairs, whereas a bold value illustrates the average performance. A bold italic value indicates the excellent performance of a feature in separating class pairs that equates to a nearly complete separation of class pairs. The values of DF for all class pairs were quite varied, depending on the feature. In general, the DF derived by the fully polarimetric data tends to have a higher value than those derived by the dual-polarization data, particularly for class pairs 1 to 4 . However, for the class pair 5 , the DF derived by the fully polarimetric data was inferior to those derived by the dual-polarization data. In addition, the quality of DF for class pairs 4 and 5 generated by all polarimetric features was low. The low performance of DF occurred due to the highly overlapping mean and standard deviation values of land use/cover classes in the associated class pairs that corresponded to the stage of identifying the existence of peatland in a land use/cover class.

\subsection{Selected Features for Classification}

\subsubsection{Data combination (i)}

Table 4 shows the list of selected features for each combination of data. For dual-polarization data, four polarimetric features-dFBD_HV, wFBD_HV, wFBD_HH, and dFBD_HH-were selected in separating class pair 1. For class pair 2, two polarimetric features were selected, 
Novresiandi and Nagasawa: Polarimetric synthetic aperture radar application for tropical...

Table 3 Separability of five class pairs by each polarimetric feature.

\begin{tabular}{|c|c|c|c|c|c|c|}
\hline \multirow[b]{2}{*}{ Source } & \multirow[b]{2}{*}{ Code name } & \multicolumn{5}{|c|}{ Df of each class pair } \\
\hline & & 1 & 2 & 3 & 4 & 5 \\
\hline \multirow[t]{20}{*}{ Fully polarimetric data } & PLR_HH & 0.73 & 2.30 & 0.58 & 0.32 & 0.19 \\
\hline & PLR_HV & 1.27 & 1.68 & 0.97 & 0.22 & 0.07 \\
\hline & PLR_VH & 1.26 & 1.68 & 0.94 & 0.19 & 0.13 \\
\hline & PLR_VV & 0.77 & 2.37 & 0.27 & 0.32 & 0.18 \\
\hline & PLR_H & 0.53 & 1.27 & 1.03 & 0.10 & 0.10 \\
\hline & PLR_A & 1.79 & 0.47 & 0.58 & 0.03 & 0.11 \\
\hline & PLR_ $\alpha$ & 0.15 & 1.73 & 0.76 & 0.12 & 0.15 \\
\hline & f_odd & 0.10 & 2.47 & 0.22 & 0.01 & 0.06 \\
\hline & f_double & 0.07 & 0.37 & 0.09 & 0.00 & 0.06 \\
\hline & f_volume & 1.36 & 2.09 & 0.69 & 0.26 & 0.17 \\
\hline & y_odd & 0.22 & 2.62 & 0.43 & 0.07 & 0.01 \\
\hline & y_double & 0.01 & 0.99 & 0.14 & 0.01 & 0.09 \\
\hline & y_volume & 1.38 & 2.09 & 0.74 & 0.23 & 0.15 \\
\hline & y_helix & 0.70 & 1.24 & 0.12 & 0.18 & 0.08 \\
\hline & SPAN & 1.02 & 2.96 & 0.02 & 0.16 & 0.20 \\
\hline & $\mathrm{HH}+\mathrm{VV}$ & 0.76 & 2.41 & 0.45 & 0.34 & 0.21 \\
\hline & $\mathrm{HH}-\mathrm{VV}$ & 0.10 & 0.11 & 0.45 & 0.01 & 0.04 \\
\hline & $2 \mathrm{HV}$ & 1.27 & 1.68 & 0.97 & 0.22 & 0.07 \\
\hline & $\mathrm{HV} / \mathrm{HH}$ & 0.03 & 0.42 & 0.08 & 0.06 & 0.22 \\
\hline & RVI & 0.37 & 1.12 & 1.80 & 0.24 & 0.20 \\
\hline \multirow[t]{5}{*}{ Dry-scene dual-polarization data } & dFBD_HH & 0.92 & 1.65 & 0.04 & 0.20 & 0.18 \\
\hline & dFBD_HV & 1.30 & 1.35 & 0.58 & 0.24 & 0.15 \\
\hline & dFBD_H & 0.35 & 0.93 & 0.63 & 0.07 & 0.02 \\
\hline & dFBD_A & 0.31 & 0.96 & 0.65 & 0.09 & 0.00 \\
\hline & $\mathrm{dFBD} \alpha$ & 0.23 & 0.85 & 0.62 & 0.08 & 0.00 \\
\hline \multirow[t]{5}{*}{ Wet-scene dual-polarization data } & wFBD_HH & 0.94 & 1.80 & 0.05 & 0.26 & 0.29 \\
\hline & wFBD_HV & 1.26 & 1.63 & 0.61 & 0.23 & 0.22 \\
\hline & wFBD_H & 0.32 & 1.18 & 0.59 & 0.02 & 0.04 \\
\hline & wFBD_A & 0.27 & 1.25 & 0.60 & 0.01 & 0.04 \\
\hline & wFBD $\alpha$ & 0.23 & 1.13 & 0.58 & 0.00 & 0.05 \\
\hline
\end{tabular}

namely, wFBD_HH and dFBD_HH. A total of four polarimetric features were selected in separating class pair 3, i.e., dFBD_A, dFBD_H, wFBD_HV, and wFBD_A. In separating class pair 4, wFBD_HH and dFBD_HV were selected. For class pair 5, wFBD_HH and wFBD_HV were selected in the algorithm of the decision tree for tropical peatlands classification. Thus, the polarimetric features chosen for dual-polarization data were mainly derived by the backscatter 
Novresiandi and Nagasawa: Polarimetric synthetic aperture radar application for tropical...

Table 4 List of selected polarimetric features for each data combination.

\begin{tabular}{|c|c|c|c|c|}
\hline \multirow[b]{2}{*}{ Class pair } & \multicolumn{4}{|c|}{ Combinations of data } \\
\hline & $\mathrm{i}$ & ii & iii & iv \\
\hline 1 & $\begin{array}{l}\text { dFBD_HV, wFBD_HV, } \\
\text { wFBD_HH, and dFBD_HH }\end{array}$ & $\begin{array}{l}\text { y_volume and } \\
\text { f_volume }\end{array}$ & $\begin{array}{l}\text { y_volume and } \\
\text { f_volume }\end{array}$ & $\begin{array}{l}\text { y_volume and } \\
\text { f_volume }\end{array}$ \\
\hline 2 & wFBD_HH and dFBD_HH & SPAN and y_odd & SPAN and y_odd & SPAN and y_odd \\
\hline 3 & $\begin{array}{l}\text { dFBD_A, dFBD_H, } \\
\text { wFBD_HV, and wFBD_A }\end{array}$ & $\mathrm{RVI}$ & RVI & RVI \\
\hline 4 & wFBD_HH and dFBD_HV & $\begin{array}{l}\mathrm{HH}+\mathrm{VV}, \mathrm{PLR} \mathrm{HH}, \\
\text { PLR_VV, and } \\
\text { f_volume }\end{array}$ & $\begin{array}{l}\text { HH+VV, PLR_HH, } \\
\text { PLR_VV, f_volume, } \\
\text { wFBD_HH and } \\
\text { dFBD_HV }\end{array}$ & $\begin{array}{l}\mathrm{HH}+\mathrm{VV}, \mathrm{PLR} \text { _HH, } \\
\text { PLR_VV, f_volume, } \\
\text { wFBD_HH, dFBD_HV, } \\
\text { and "distance to river" }\end{array}$ \\
\hline 5 & wFBD_HH and wFBD_HV & $\begin{array}{l}\mathrm{HH}+\mathrm{VV}, \mathrm{RVI} \\
\text { and SPAN }\end{array}$ & $\begin{array}{l}\text { wFBD_HH and } \\
\mathrm{HV} / \mathrm{HH}\end{array}$ & $\begin{array}{l}\text { wFBD_HH, } \mathrm{HV} / \mathrm{HH} \text {, } \\
\text { and "distance to river" }\end{array}$ \\
\hline
\end{tabular}

coefficient, whereas those derived by Cloude-Pottier decomposition were selected only in class pair 3. This shows that the backscatter coefficient of the dual-polarization data was dominant among almost all land use/cover classes described in this study for the purpose of tropical peatlands classification.

Moreover, selected features in separating class pairs 4 and 5 described important characteristics in identifying the existence of peatland as well as in understanding the seasonal variation of tropical peatlands, especially from the viewpoint of L-band SAR dual-polarization data. For class pair 4, in separating the classes "bareland with peat" and "bareland without peat," both features derived by the dry- and wet-scene dual-polarization data were used. Nevertheless, only features derived by the wet-scene dual-polarization data were selected to separate "vegetation with peat" and "vegetation without peat" classes. These characteristics were obviously seen by the value of DF, as shown in Table 3, whereby the DF of selected features for class pair 4 derived by dry- and wet-scene dual-polarization data was relatively high. However, for class pair 5 , the DF of selected features derived by the wet-scene dual-polarization data was higher than the dry scene. Therefore, the existence of peatland in a land use/cover without the presence of vegetation, such as that in bareland, was not influenced by the seasonal condition as it generates a relatively similar response to backscatter coefficients derived by the wet- and dry-scene dual-polarization data. On the other hand, the existence of peatland in a land use/cover with the presence of vegetation (or below a vegetation cover) was influenced by the seasonal condition, whereby it was more sensitive to backscatter coefficients derived by the wet-scene dualpolarization data that was taken during the wet season. This finding was valuable in increasing the state of knowledge of tropical peatlands for classification, especially from the viewpoint of L-band dual-polarization SAR data.

\subsubsection{Data combination (ii)}

Regarding the fully polarimetric data, two polarimetric features were selected in separating class pair 1, y_volume and f_volume. To separate class pair 2, SPAN and y_odd were selected. On the other hand, the feature RVI was selected to separate class pair 3. A total of four features were selected in separating class pair 4, i.e., HH+VV, PLR_HH, PLR_VV, and f_volume. In separating class pair 5, three features- $\mathrm{HH}+\mathrm{VV}$, RVI, and SPAN-were selected in the algorithm for tropical peatlands classification. Therefore, the selected polarimetric features for fully polarimetric data showed good complementarity between features derived by Freeman-Durden threecomponent decomposition, Yamaguchi four-component decomposition, the RVI, the total scattering power, and the copolarized backscatter coefficient measurements. However, polarimetric features derived by Cloude-Pottier decomposition and cross-polarized backscatter coefficient 
measurements were not selected due to their low performance in separating class pairs, as they did not have any effect on increasing the accuracy of the classification results. This indicated that the fully polarimetric data had the potential to characterize land use/cover classes described in this study for the purpose of tropical peatlands classification.

\subsubsection{Data combination (iii)}

For the combination of dual-polarization and fully polarimetric data, the selected polarimetric features in separating the first three class pairs (class pairs 1,2, and 3) were the same features as those selected in the first three class pairs for the fully polarimetric data. Thus, two polarimetric features-y_volume and f_volume-were selected in separating class pair 1. For class pair 2, two polarimetric features were selected, namely, SPAN and y_odd. In separating class pair 3, the feature RVI was selected. However, to separate class pair 4, six features were selected, HH+VV, PLR_HH, PLR_VV, f_volume, wFBD_HH, and dFBD_HV. For class pair 5, wFBD_HH and the depolarization ratio were selected in the algorithm of the decision tree for tropical peatlands classification.

Thus, the selected polarimetric features for the combination of dual-polarization and fully polarimetric data indicated a dominance of features derived by the fully polarimetric data, especially those selected in the first three class pairs. Polarimetric features derived by the dual-polarization data were mainly selected in separating class pairs 4 and 5 , which was the stage of identifying the existence of peatland in a land use/cover class. This showed that the dual-polarization data aided the fully polarimetric data in characterizing the existence of peatland in a land use/cover class.

\subsubsection{Data combination (iv)}

For the combination of dual-polarization data, fully polarimetric data, and the additional feature "distance to river," the selected polarimetric features were identical to those selected in the combination of dual-polarization and fully polarimetric data for all class pairs. The feature "distance to river" was added in separating class pairs 4 and 5. In this study, however, only the category " $<2 \mathrm{~km}$ " of the feature "distance to river" was applied to the algorithm of the decision tree classification. The integration of the additional feature "distance to river" was meant to evaluate the effectiveness of GIS data integration in the SAR-based tropical peatlands classification. Thus, in this study, the feature "distance to river" was hypothesized to produce better classification results along with the use of dual-polarization and fully polarimetric data in tropical peatlands classification.

\subsection{Results of the Image Classification}

As presented in Fig. 4, the results of tropical peatlands classification from four combinations of data were generated by means of the decision tree classifier. The classification results showed six land use/cover classes, i.e., water, artificial, bareland with peat, bareland without peat, vegetation with peat, and vegetation without peat. The "bareland with peat" and "vegetation with peat" classes represented land use/cover classes identified with the existence of peatland, whereby the "bareland without peat" and "vegetation without peat" classes represented land use/cover classes identified as having no peatland. The classification results of data combinations (i), (ii), (iii), and (iv) had 53.6\%, 40.4\%, 50.9\%, and 32.3\% of the total area identified as having peatland, respectively. The results of tropical peatlands classification derived by the dual-polarization data generated the biggest area identified as having peatland, whereas those derived by the combination of dual-polarization data, fully polarimetric data, and the additional feature "distance to river" produced the smallest area identified as having peatland. Thus, in this study, the dual-polarization data tended to generate more peatland area, whereas the full polarimetric data tended to generate less peatland area. In addition, the feature "distance to river" was added to the algorithm of decision tree classification to ignore the existence of peatland $<2 \mathrm{~km}$ from the river stream, so that it influenced the classification results of data combination (iv) producing the smallest area of peatland. 

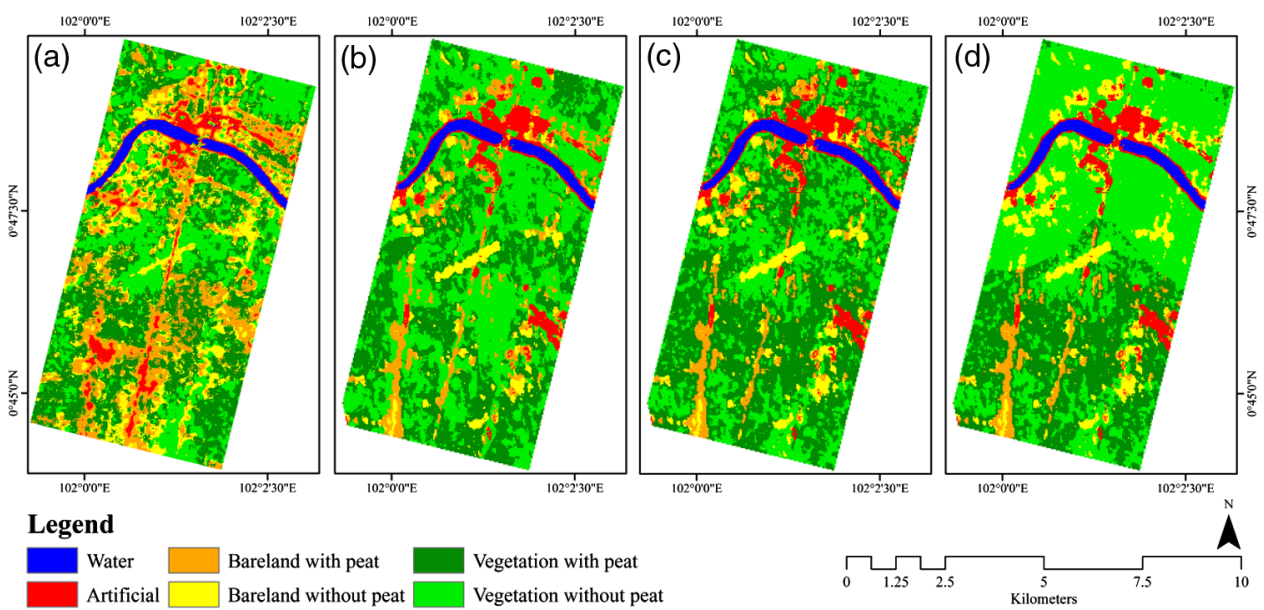

Fig. 4 Result of tropical peatlands classification for each combination of data: (a) data combination i, (b) data combination ii, (c) data combination iii, and (d) data combination iv.

\subsection{Accuracies of the Image Classification}

Table 5 shows the accuracy indicators of tropical peatlands classification results from four combinations of data by means of the decision tree classifier. Accuracy indicators were low for the classification results derived by the dual-polarization data, which also yielded the lowest $\mathrm{OA}$ and $\mathrm{K}$ of $55.0 \%$ and 0.39 , respectively. In contrast, the accuracy indicators for the classification results generated by the fully polarimetric data produced better accuracy indicators than those produced by the dual-polarization data; the fully polarimetric data gave accuracies of $65.0 \%$ and 0.51 for OA and K, respectively. Significant increases in OA and K were obtained by the classification results derived by the combination of dual-polarization and fully polarimetric data; this combination yielded an OA of $69.0 \%$ and a $\mathrm{K}$ of 0.57 . Thus, accuracy indicators were increased by applying the combination of dual-polarization and fully polarimetric data to the algorithm of decision tree classification for the SAR-based tropical peatlands classification. This combination showed that the complementarity of dual-polarization and fully polarimetric data produced higher accuracy than the single usage of each type of data for the purpose of tropical peatlands classification by means of the decision tree classifier. In addition, the best accuracy was obtained by means of integrating the feature "distance to river" into the algorithm of decision tree classification. The accuracy indicators derived by the combination of

Table 5 Accuracy indicators for tropical peatlands classification for each combination of data.

\begin{tabular}{|c|c|c|c|c|c|c|c|c|}
\hline \multirow[b]{3}{*}{ Class name } & \multicolumn{8}{|c|}{ Combinations of data } \\
\hline & \multicolumn{2}{|c|}{$\mathrm{i}$} & \multicolumn{2}{|c|}{ ii } & \multicolumn{2}{|c|}{ iii } & \multicolumn{2}{|c|}{ iv } \\
\hline & PA (\%) & UA (\%) & PA (\%) & UA (\%) & PA (\%) & UA (\%) & PA (\%) & UA (\%) \\
\hline Water & 100.0 & 100.0 & 100.0 & 100.0 & 100.0 & 100.0 & 100.0 & 100.0 \\
\hline Artificial & 44.4 & 55.2 & 66.7 & 85.7 & 66.7 & 85.7 & 66.7 & 85.7 \\
\hline Bareland with peat & 46.4 & 41.6 & 75.4 & 64.2 & 71.0 & 71.0 & 66.7 & 93.9 \\
\hline Bareland without peat & 52.6 & 36.0 & 62.8 & 77.8 & 71.8 & 74.7 & 93.6 & 76.8 \\
\hline Vegetation with peat & 56.7 & 52.5 & 57.1 & 60.1 & 57.1 & 64.3 & 52.9 & 83.0 \\
\hline Vegetation without peat & 53.3 & 67.3 & 66.3 & 61.1 & 75.6 & 66.2 & 92.6 & 67.9 \\
\hline $\mathrm{OA}(\%)$ & \multicolumn{2}{|c|}{55.0} & \multicolumn{2}{|c|}{65.0} & \multicolumn{2}{|c|}{69.0} & \multicolumn{2}{|c|}{76.0} \\
\hline Kappa coefficient & \multicolumn{2}{|c|}{0.39} & \multicolumn{2}{|c|}{0.51} & \multicolumn{2}{|c|}{0.57} & \multicolumn{2}{|c|}{0.66} \\
\hline
\end{tabular}


dual-polarization data, fully polarimetric data, and the additional feature "distance to river" yielded the best $\mathrm{OA}$ and $\mathrm{K}$ of $76.0 \%$ and 0.66 , respectively.

The integration of GIS data in the SAR-based tropical peatlands classification was found to be effective in improving the classification accuracy, whereas the feature "distance to river" ignored the existence of peatland $<2 \mathrm{~km}$ calculated from the main river stream. The feature "distance to river" successfully aided the polarimetric features derived by dual-polarization and fully polarimetric data in classifying tropical peatlands and increased the accuracy indicators of the classification results. The category " $<2 \mathrm{~km}$ " of the feature "distance to river" was revealed to represent the actual distance of peatland to exist as measured from the main river stream. Thus, the feature "distance to river" increased the classification accuracy when integrated with the polarimetric features derived by the combination of dual-polarization and fully polarimetric data by means of the decision tree classifier. The accuracy indicators derived by the combination of dual-polarization data, fully polarimetric data, and the additional feature "distance to river" were increased by nearly $10 \%$ for OA and $15 \%$ for K over the best obtained OA and K derived by the SAR-based tropical peatlands classification using the decision tree classifier.

\section{Conclusions}

This study evaluated the performances of PALSAR dual-polarization and fully polarimetric data for tropical peatlands classification in response to the scheme of the sustainable management of tropical peatlands, especially in the activity of mapping spatial distributions of tropical peatlands in Indonesia. There were several findings regarding the development of tropical peatlands classification using L-band SAR dual-polarization and fully polarimetric data. First, tropical peatlands is a complex ecosystem to be mapped, especially from the viewpoint of L-band SAR dual-polarization and fully polarimetric data, as they generated highly overlapping means and standard deviations with no peatland areas. Thus, in this study, a strict algorithm was successfully applied by means of the decision tree classifier to classify tropical peatlands involving the use of PALSAR dual-polarization and fully polarimetric data. Second, the seasonal variation of tropical peatlands was found, in which the existence of peatland in a land use/cover without the presence of vegetation was not influenced by the seasonal condition. In contrast, the existence of peatland in a land use/cover with the presence of vegetation (or below a vegetation cover) was influenced by the seasonal condition. Third, the classification results of the dualpolarization data were inferior to the fully polarimetric data, indicating that the fully polarimetric data were more suitable for classifying tropical peatlands. Fourth, the integration of GIS data in the SAR-based tropical peatlands classification was found to be effective in improving the classification accuracy, whereby in this study, the feature "distance to river" increased the classification accuracy when integrated with the polarimetric features derived by the combination of dual-polarization and fully polarimetric data by means of the decision tree classifier. The results and findings presented in this study could aid in improving the state of knowledge in tropical peatlands classification, especially when involving the use of L-band SAR dual-polarization and fully polarimetric data.

\section{References}

1. S. E. Page, C. J. Banks, and J. O. Rieley, "Tropical peatlands: distribution, extent and carbon storage-uncertainties and knowledge gaps," Peatlands Int. 2, 26-27 (2007).

2. S. E. Page, J. O. Rieley, and C. J. Banks, "Global and regional importance of tropical peatland carbon pool," Global Change Biol. 17(2), 798-818 (2011).

3. S. Shimada, M. Takada, and H. Takahashi, "Peat mapping," in Tropical Peatland Ecosystems, M. Osaki and N. Tsuji, Ed., pp. 455-467, Springer, Tokyo, Japan (2016).

4. B. Rudiyanto et al., "Digital mapping for cost-effective and accurate prediction of the depth and carbon stocks in Indonesian peatlands," Geoderma 272, 20-31 (2016).

5. D. Lu, "The potential and challenge of remote sensing-based biomass estimation," Int. J. Remote Sens. 27(7), 1297-1328 (2006).

6. S. Kuntz, "Potential of spaceborne SAR for monitoring the tropical environments," Trop. Ecol. 51(1), 3-10 (2010). 
Novresiandi and Nagasawa: Polarimetric synthetic aperture radar application for tropical...

7. O. Antropov et al., "Peatland delineation under forest canopy with polsar data using model based decomposition technique," in Proc. IEEE Int. Geoscience and Remote Sensing Symp., pp. 4918-4921 (2012).

8. O. Antropov et al., "Land cover and soil type mapping from spaceborne PolSAR data at L-band with probabilistic neural network," IEEE Trans. Geosci. Remote Sens. 52(9), 5256-5270 (2014).

9. A. Wijaya, P. R. Marpu, and R. Gloaguen, "Discrimination of peatlands in tropical swamp forests using dual-polarimetric SAR and Landsat ETM data," Int. J. Image Data Fusion 1(3), 257-270 (2010).

10. M. Watanabe et al., "PALSAR full-polarimetric observation for peatland," Asian J. Geoinf. 11(3), (2011).

11. G. C. Dargie et al., "Age, extent and carbon storage of the central Congo Basin peatland complex," Nature 542(2), 86-90 (2017).

12. M. Takada, Y. Mishima, and S. Natsume, "Estimation of surface soil properties in peatland using ALOS/PALSAR," Landscape Ecol. Eng. 5(1), 45-58 (2009).

13. O. Antropov, Y. Rauste, and T. Häme, "Volume scattering modeling in PolSAR decompositions: study of ALOS PALSAR data over boreal forest," IEEE Trans. Geosci. Remote Sens. 49(10), 3838-3848 (2011).

14. S. Sabiham and S. Kartawisastra, "Peatland management for oil palm development in indonesia," Indones. J. Land Resour. 6(2), 55-66 (2012).

15. S. R. Cloude and E. Pottier, "A review of target decomposition theorems in radar polarimetry," IEEE Trans. Geosci. Remote Sens. 34(2), 498-518 (1996).

16. A. Freeman and A. L. Durden, "A three-component scattering model for polarimetric SAR data," IEEE Trans. Geosci. Remote Sens. 36, 963-973 (1998).

17. Y. Yamaguchi et al., "Four-component scattering model for polarimetric SAR image decomposition," IEEE Trans. Geosci. Remote Sens. 43(8), 1699-1706 (2005).

18. J. S. Lee and E. Pottier, Polarimetric Radar Imaging: From Basics to Applications, CRC Press Taylor and Francis Group, Florida (2009).

19. Y. Kim and J. Van Zyl, "Vegetation effects on soil moisture estimation," in Proc. Int. Geoscience and Remote Sensing Symp., pp. 800-802, IEEE International, Anchorage (2004).

20. A. Hooijer and R. Vernimmen, Peatland Maps: Accuracy Assessment and Recommendations (TA-QANS), Deltares \& Euroconsult Mott MacDonald, Netherlands (2013).

21. S. Shimada, H. Takahashi, and M. Osaki, "Carbon stock estimate," in Tropical Peatland Ecosystems, M. Osaki and N. Tsuji, Ed., pp. 353-365, Springer, Tokyo, Japan (2016).

22. M. A. Friedl and C. E. Brodley, "Decision tree classification of land cover from remotely sensed data," Remote Sens. Environ. 61, 399-409 (1997).

23. M. Pal and P. M. Mather, "An assessment of the effectiveness of decision tree methods for land cover classification," Remote Sens. Environ. 86, 554-565 (2003).

24. P. Mishra, D. Singh, and Y. Yamaguchi, "Land cover classification of Palsar images by knowledge based decision tree classifier and supervised classifiers based on SAR observables," Prog. Electromagn. Res. B 30, 47-70 (2011).

25. I. G. Cumming and J. J. Van Zyl, "Feature utility in polarimetric radar image classification," in Proc. IEEE Int. Geoscience and Remote Sensing Symp. (IGARSS), pp. 1841-1846 (1989).

26. J. Chen, H. Lin, and Z. Pei, "Application of ENVISAT ASAR data in mapping rice crop growth in southern China," IEEE Geosci. Remote Sens. Lett. 4(3), 431-435 (2007).

Dandy Aditya Novresiandi is currently working toward his $\mathrm{PhD}$ at the United Graduate School of Agricultural Sciences (USGAS), Tottori University, Japan. His research interests lie in optical and radar remote sensing of environment and agriculture.

Ryota Nagasawa is currently a professor with the Faculty of Agriculture, Tottori University, Japan. His research activities cover the applications of remote sensing and geographic information systems for landscape ecology. 